\title{
Clinical predictors of lacunar syndrome not due to lacunar infarction
}

\author{
Adrià Arboix*1, Joan Massons'1 , Luis García-Eroles², Cecilia Targa', Emili Comes and Olga Parra3,4
}

\begin{abstract}
Background
Lacunar syndrome not due to lacunar infarct is poorly characterised. This single centre, retrospective study was conducted to describe the clinical characteristics of patients with lacunar syndrome not due to lacunar infarct and to identify clinical predictors of this variant of lacunar stroke.

Methods: A total of 146 patients with lacunar syndrome not due to lacunar infarction were included in the "Sagrat Cor Hospital of Barcelona Stroke Registry" during a period of 19 years (1986-2004). Data from stroke patients are entered in the stroke registry following a standardized protocol with 161 items regarding demographics, risk factors, clinical features, laboratory and neuroimaging data, complications and outcome. The characteristics of these 146 patients with lacunar syndrome not due to lacunar infarct were compared with those of the 733 patients with lacunar infarction.
\end{abstract}

Results: Lacunar syndrome not due to lacunar infarct accounted for $16.6 \%$ (146/879) of all cases of lacunar stroke. Subtypes of lacunar syndromes included pure motor stroke in 63 patients, sensorimotor stroke in 51, pure sensory stroke in 14, atypical lacunar syndrome in 9, ataxic hemiparesis in 5 and dysarthria-clumsy hand in 4 . Valvular heart disease, atrial fibrillation, sudden onset, limb weakness and sensory symptoms were significantly more frequent among patients with lacunar syndrome not due to lacunar infarct than in those with lacunar infarction, whereas diabetes was less frequent. In the multivariate analysis, atrial fibrillation $(O R=4.62)$, sensorimotor stroke $(O R=4.05)$, limb weakness $(\mathrm{OR}=2.09)$, sudden onset $(\mathrm{OR}=2.06)$ and age $(\mathrm{OR}=0.96)$ were independent predictors of lacunar syndrome not due to lacunar infarct.

Conclusions: Although lacunar syndromes are highly suggestive of small deep cerebral infarctions, lacunar syndromes not due to lacunar infarcts are found in $16.6 \%$ of cases. The presence of sensorimotor stroke, limb weakness and sudden onset in a patient with atrial fibrillation should alert the clinician to the possibility of a lacunar syndrome not due to a lacunar infarct.

\section{Background}

Lacunar syndromes are usually caused by lacunar infarctions [1,2]. However, other stroke subtypes, including small intracerebral haemorrhages, spontaneous subdural haematomas or non-lacunar cerebral infarctions may occasionally be the aetiology of lacunar syndromes. Lacunar syndromes not due to lacunar infarcts are poorly defined [3-5]. Therefore, the aim of this study was to describe the clinical characteristics of patients with lacunar syndrome not due to lacunar infarct and to identify clinical predictors of this variant of lacunar stroke. As far as we are aware, the present series of patients with lacu-

* Correspondence: aarboix@hscor.com

1 Unit of Cerebrovascular Diseases, Service of Neurology, Hospital Universitari del Sagrat Cor, Universitat de Barcelona, Barcelona, Spain

Full list of author information is available at the end of the article nar syndrome not due to lacunar infarct collected from a prospective stroke registry represents the largest experience of this infrequent stroke subtype reported in the literature.

\section{Methods}

The database of the "Sagrat Cor Hospital of Barcelona Stroke Registry" with data of 3808 acute stroke patients was searched for those with a diagnosis of first-ever lacunar stroke who were admitted consecutively to the Department of Neurology of the Sagrat Cor Hospital (an acute-care 350-bed teaching hospital in the city of Barcelona) between January 1986 and December 2004. Details of this on-going hospital-based stroke registry have been previously reported [6]. Data from stroke patients are entered following a standardised protocol with 161 items 
regarding demographics, risk factors, clinical features, laboratory and neuroimaging data, complications and outcome. Subtypes of stroke were classified according to the Cerebrovascular Study Group of the Spanish Society of Neurology, which is similar to the National Institute of Neurological Disorders and Stroke classification [7].

For the purpose of this study, all cases of first-ever lacunar stroke diagnosed in 879 patients were collected. Lacunar infarcts were defined $[6,8]$ as $(a)$ sudden or gradual onset of a focal neurological deficit lasting $>24$ hours of the type described in the common lacunar syndromes (pure motor hemiparesis, pure sensory stroke, sensorimotor stroke, ataxic hemiparesis, dysarthria-clumsy hand and atypical lacunar syndromes); (b) brain CT scans or MRI were either normal or demonstrated only small, localised brain lesions with diameter smaller than $20 \mathrm{~mm}$ that seemed appropriate for the neurological deficits, and (c) absence of cortical ischaemia, cervical carotid stenosis (> 50\% diameter) or major source for cardioembolic stroke. Lacunar syndromes not due to lacunar infarcts were defined as sudden or gradual onset of a focal neurological deficit lasting $>24$ hours of the type described in the common lacunar syndrome (pure motor hemiparesis, pure sensory stroke, sensorimotor stroke, ataxic hemiparesis, dysarthria-clumsy hand and atypical lacunar syndrome[8]) secondary to non-lacunar ischaemic stroke (cortical or subcortical lesions $>20 \mathrm{~mm}$ with atherothrombotic, cardioembolic, unusual etiology or unknown etiology) or haemorrhagic stroke (intracerebral haemorrhage or spontaneous subdural haematoma).

All patients were admitted to the hospital within 48 hours of the onset of symptoms. Systematic investigations included clinical examination, 12-lead electrocardiogram, chest radiology, and standard blood tests including serum cholesterol concentration and lipid profile. A brain CT scan and/or MRI (41\%) was performed within this first week of hospital admission. Angio-MRI was performed in $38 \%$ of the patients. Other investigations included Doppler ultrasonography of the suparaaortic trunks in $65.5 \%$ of the cases, arterial digital subtraction angiography in 8\%, 2-dimensional echocardiography in $41.2 \%$, and lumbar puncture in $2.8 \%$. Demographic data, vascular risk factors, clinical features, neuroimaging findings, and outcome were recorded. Details of variables prospectively registered in the database have been previously reported [6]. Medical complications (respiratory, urinary, cardiac, vascular and infectious) and mortality during the acute phase of the disease was assessed. Causes of death were analyzed according to criteria of Silver et al. [9].

\section{Statistical analysis}

Demographic characteristics, risk factors, aetiology of stroke, subtypes of stroke and of lacunar syndromes, clin- ical events and outcome of patients with lacunar syndrome not due to lacunar infarct and those with lacunar infarction were compared with the analysis of variance (ANOVA) (with Bonferroni's correction when necessary) and the chi-square $\left(X^{2}\right)$ test (with Yate's correction when necessary). Variables were subjected to multivariate analysis with a logistic regression procedure and forward stepwise selection if $P<0.10$ after univariate testing. The effect of variables on the presence of a lacunar syndrome not due to lacunar infarct was analysed in a predictive model based on demographic, vascular risk factors and clinical, variables, in which lacunar syndrome not due to lacunar infarct was the dependent variable. Age was used as a continuous variable with a constant odds ratio for each year. The odds ratio (OR) and 95\% confidence intervals $(\mathrm{CI})$ were calculated from the beta coefficients and standard errors. Statistical significance was set at $P<$ 0.05 .

\section{Results}

The diagnosis of lacunar syndrome not due to lacunar infarct was established in 146 (16.6\%) of all 879 consecutive patients with first-ever lacunar stroke. There were 82 men and 64 women with a mean (SD) age of 71.6 (12.6) years. Twenty-six patients aged 85 years or older. The following vascular risk factors in a decreasing order of frequency were observed: hypertension (73.3\%), atrial fibrillation (30.1\%), diabetes mellitus (21.2\%), hyperlipidemia (19.9\%), ischaemic heart disease (15.8\%) and heavy smoking (> 20 cigarettes/day) (13\%). As shown in Table 1, subtypes of lacunar syndromes not due to lacunar stroke included pure motor stroke in $43 \%(63 / 146)$ of patients, sensorimotor stroke in 35\% (52/146), pure sensory stroke in $9.5 \%(14 / 146)$, atypical lacunar syndrome in $6.2 \%(9 /$ 146), ataxic hemiparesis in $3.5 \%(5 / 146)$ and dysarthriaclumsy hand in $2.7 \%(4 / 146)$. In relation to the total number of patients with the different subtypes of lacunar syndromes, those not due to lacunar stroke accounted for $15.2 \%(63 / 415)$ of pure motor strokes, $38.8 \%$ (52/143) of sensorimotor strokes, $9.9 \%(14 / 141)$ of pure sensory strokes, $9.3 \%$ (9/27) of atypical lacunar syndromes, $17.2 \%$ $(5 / 29)$ of ataxic hemiparesis and $6.3 \%(4 / 63)$ of dysarthria-clumsy hand.

In relation to the aetiology of the different stroke subtypes included in the database, lacunar syndrome not due to lacunar infarct occurred in $10.5 \%(4 / 38)$ of cases of spontaneous subdural haematoma, 9.1\% (37/408) of primary intracerebral haemorrhage, 7\% (54/770) of atherothrombotic infarction, 5\% (38/763) of cardioembolic infarction, $3.4 \%(11 / 324)$ of infarcts of unknown cause and $1.8 \%(2 / 114)$ of infarcts of unusual aetiology.

One patient died (in-hospital mortality rate $0.7 \%$ ). This patient died suddenly of an unknown cause. The median length of hospitalization was 12 days (interquartile range 
Table 1: Results of univariate analysis in patients with lacunar syndrome not due to lacunar infarction compared with patients with lacunar infarct

\begin{tabular}{|c|c|c|c|}
\hline Data & $\begin{array}{l}\text { Lacunar syndrome not due } \\
\text { to lacunar infarct }\end{array}$ & Lacunar infarct & $P$ value \\
\hline Total patients & 146 & 733 & \\
\hline Males & $82(56.2)$ & $423(57.7)$ & 0.730 \\
\hline Age, years, mean (SD) & $72.9(12.6)$ & $74.1(10.2)$ & 0.285 \\
\hline Age $\geq 85$ years & $26(17.8)$ & $110(15.0)$ & 0.393 \\
\hline \multicolumn{4}{|l|}{ Cardiovascular risk factors } \\
\hline Hypertension & $107(73.3)$ & $525(71.6)$ & 0.683 \\
\hline Diabetes mellitus & $31(21.2)$ & $218(29.7)$ & 0.037 \\
\hline Valvular heart disease & $10(6.8)$ & $21(2.9)$ & 0.017 \\
\hline Ischaemic heart disease & $23(15.8)$ & $104(14.2)$ & 0.623 \\
\hline Atrial fibrillation & $44(30.1)$ & $81(11.1)$ & 0.0001 \\
\hline Congestive heart failure & $4(2.7)$ & $24(3.3)$ & 0.737 \\
\hline $\begin{array}{l}\text { Transient ischaemic } \\
\text { attack }\end{array}$ & $12(8.2)$ & $80(10.9)$ & 0.331 \\
\hline $\begin{array}{l}\text { Previous cerebral } \\
\text { infarction }\end{array}$ & $16(11)$ & $117(16)$ & 0.123 \\
\hline Periphery arterial disease & $17(11.6)$ & $57(7.8)$ & 0.124 \\
\hline Obesity & $8(5.5)$ & $47(6.4)$ & 0.671 \\
\hline $\begin{array}{l}\text { Alcohol abuse (> } 80 \mathrm{~g} / \\
\text { day) }\end{array}$ & $7(4.8)$ & $21(2.9)$ & 0.340 \\
\hline $\begin{array}{l}\text { Heavy smoking (> } 20 \\
\text { cigarettes/day) }\end{array}$ & $19(13)$ & $86(11.7)$ & 0.663 \\
\hline Hyperlipidemia & 29 (19.9) & $166(22.6)$ & 0.460 \\
\hline \multicolumn{4}{|l|}{ Clinical features } \\
\hline Sudden onset (minutes) & $78(53.4)$ & $310(42.3)$ & 0.013 \\
\hline Headache & $15(0.3)$ & $68(9.3)$ & 0.707 \\
\hline Early seizures & $1(0.7)$ & 0 & 0.369 \\
\hline Limb weakness & $129(88.4)$ & $554(75.6)$ & 0.001 \\
\hline Sensory symptoms & $64(43.8)$ & $231(31.5)$ & 0.004 \\
\hline Speech disturbances & $52(35.6)$ & $311(42.4)$ & 0.127 \\
\hline Ataxia & $8(5.5)$ & $50(6.8)$ & 0.551 \\
\hline Cranial nerve palsy & $4(2.7)$ & $21(2.9)$ & 1 \\
\hline Lacunar syndromes & & & 0.0001 \\
\hline Pure motor hemiparesis & $63(43)$ & $352(48)$ & \\
\hline Pure sensory stroke & $14(9.5)$ & $127(17.3)$ & \\
\hline Sensorimotor stroke & $51(35)$ & $83(11.3)$ & \\
\hline Dysarthria-clumsy hand & $4(2.7)$ & $59(8)$ & \\
\hline Ataxic hemiparesis & $5(3.5)$ & $24(3.3)$ & \\
\hline $\begin{array}{l}\text { Atypical lacunar } \\
\text { syndrome* }\end{array}$ & $9(6.2)$ & $88(12)$ & \\
\hline \multicolumn{4}{|l|}{ Outcome } \\
\hline $\begin{array}{l}\text { Symptom-free at } \\
\text { discharge }\end{array}$ & $27(18.5)$ & $166(22.6)$ & 0.268 \\
\hline
\end{tabular}


Table 1: Results of univariate analysis in patients with lacunar syndrome not due to lacunar infarction compared with patients with lacunar infarct (Continued)

\begin{tabular}{lccc}
\hline Respiratory events & $5(3.4)$ & $17(2.3)$ & 0.624 \\
Urinary infection & $7(4.8)$ & $20(2.7)$ & 0.290 \\
Cardiac events & $5(3.4)$ & $8(1.1)$ & 0.079 \\
Vascular complications & $1(0.7)$ & $4(0.5)$ & 1 \\
$\quad$ Infectious complications & $7(4.8)$ & $28(3.8)$ & 0.582 \\
Length of hospital stay, days, & $12(8.5-20)$ & $10(7-14)$ & 0.001 \\
(median, interquartile range & & & 1 \\
[25th-75th percentile) & $1(0.7)$ & $4(0.5)$ &
\end{tabular}

Data are $n$ (\%) unless otherwise stated.

*Dysarthria facial paresis in 3 patients, isolated dysarthria in 2, bilateral pure motor hemiparesis with transient subcortical aphasia in 2, pure motor hemiparesis with transient internuclear ophthalmoplegia in 1, and paramedian thalamic infarct syndrome in 1.

8.5-20 days). Only 27 (18.5\%) patients were symptom-free at the time of hospital discharge.

As shown in Table 1, valvular heart disease, atrial fibrillation, sudden onset, limb weakness and sensory symptoms were significantly more frequent among patients with lacunar syndrome not due to lacunar infarct than in those with lacunar infarction, whereas diabetes was less frequent. In the multivariate analysis, atrial fibrillation $(\mathrm{OR}=4.62)$, sensorimotor stroke $(\mathrm{OR}=4.05)$, limb weakness $(\mathrm{OR}=2.09)$, sudden onset $(\mathrm{OR}=2.06)$ and age $(\mathrm{OR}=$ $0.96)$ were independent predictors of lacunar syndrome not due to lacunar infarct (Table 2).

\section{Discussion}

In the present series, the incidence of lacunar syndrome not due to lacunar infarct was $16.6 \%$. In earlier studies, the frequency of acute lacunar syndromes varied between $6 \%$ and $42 \%[3,14,15]$ but these series studied patients with pure motor stroke only [10] or included patients with non-vascular aetiologies (multiple sclerosis, cerebral abscess, glioblastoma) [3,10]. In small series of patients, studies in which MRI examination was performed during the acute phase of stroke, it was observed that lacunar syndromes were predictors of lacunar infarcts [11-13]. In one of these studies [12], cortical involvement was found in only 2 out of 23 patients. In another study [13], acute diffusion-weighted MRI altered the final diagnosis of small vessel occlusion in $40 \%$ of 73 patients with a lacunar syndrome [13]. Most of these 'false positive' cases are attributable to large artery or cardiogenic embolic stroke. Therefore, the present results based on a clinical series of 146 consecutive patients diagnosed of lacunar syndrome not due to lacunar infarct, --the largest reported in the literature up to the present time--, reinforce the lacunar hypothesis, which states that in the presence of a lacunar

Table 2: Variables associated with lacunar syndrome not due to lacunar infarct

\begin{tabular}{|c|c|c|c|c|}
\hline Variable & $\boldsymbol{\beta}$ & SE ( $\beta$ ) & Odds ratio $(95 \% \mathrm{CI})$ & $p$ \\
\hline \multicolumn{5}{|l|}{$\begin{array}{l}\text { Model based on } \\
\text { demographics, } \\
\text { vascular risk factors } \\
\text { and clinical variables* }\end{array}$} \\
\hline Atrial fibrillation & 1.532 & 0.301 & $4.62(2.56-8.36)$ & 0.0001 \\
\hline $\begin{array}{l}\text { Sensorimotor } \\
\text { stroke }\end{array}$ & 1.396 & 0.294 & 4.05 (2.28-7.19) & 0.0001 \\
\hline Limb weakness & 0.738 & 0.363 & $2.09(1.03-4.26)$ & 0.042 \\
\hline Sudden onset & 0.721 & 0.253 & $2.06(1.25-3.37)$ & 0.004 \\
\hline Age & -0.038 & 0.011 & $0.96(0.94-0.98)$ & 0.001 \\
\hline
\end{tabular}

${ }^{*} \beta=-0.521 ;$ SE $(\beta)=0.825 ;$ goodness-of-fit $\times^{2}=3.675 ; d f=8 ; P=0.885 ;$ area under the ROC curve $=0.753 ;$ sensitivity $67 \%$; specificity $74 \%$; positive predictive value $33 \%$; negative predictive value $92 \%$; correct classification $73 \%$. 
syndrome defined only by clinical data, lacunar infarction is the most common aetiology [4].

In relation to aetiological stroke subtypes, lacunar syndrome not due to lacunar infarct was found in $10.5 \%$ of cases of spontaneous subdural haematoma and in $9.1 \%$ of primary intracerebral haemorrhage. Subdural haematomas and intracerebral haemorrhage have been reported to cause non-ischaemic lacunar syndromes not due to lacunar infarct $[3,14]$. Likewise, lacunar syndromes not due to lacunar infarcts were documented in $7 \%$ of atherothrombotic infarction, $5 \%$ of cardioembolic infarction, $3.4 \%$ of infarcts of unknown cause and $1.8 \%$ of infarcts of unusual aetiology, which is in agreement with other studies showing that lacunar syndromes may be caused by extensive non-lacunar infarctions, mainly of subcortical topography and less frequently cortical infracts $[3,13]$.

A sensorimotor stroke is possible from a single lacune and constitutes one of the five classic or typical lacunar syndromes [15-17]. However, there has been some disagreement whether sensorimotor stroke should be included among the lacunar syndromes, mainly because of limited pathological confirmation [16]. In our study, sensorimotor stroke may not be caused by a lacunar infarct in $38.8 \%$ of cases. In addition, sensorimotor stroke was an independent predictor of lacunar syndrome mot caused by lacunar infarct in the logistic regression model. These results are consistent with the study of Staaf et al. [18] in which $31 \%$ of patients with sensorimotor stroke had a potential cardioembolic source, large artery disease or infarcts not compatible with perforating artery disease. Therefore, in about one in three patients with sensorimotor stroke, aetiologies other than ischaemic small vessel disease may be present.

Atrial fibrillation was also independently associated with lacunar syndrome not due to lacunar infarct. This finding agrees with other studies showing that atrial fibrillation is less frequent in lacunar infarcts than in other non-lacunar strokes $[5,19]$ and that patients with a lacunar syndrome but a territorial infarct more often had a cardioembolic source [5]. Cardioembolism constitutes a rare cause for acute lacunar infarcts [3]. Emboligenous cardiopathy as the only demonstrable cause has been found in $2.6-5 \%$ of lacunar infarctions [20,21] and its role in the aetiology of lacunar infarction is very rare. Macroembolism could be shown in one case originally described by Fisher and Curry related to pure motor hemiplegia [22]. In one series, it was noted that embolic cardiopathy as single aetiology was only seen in $4 \%$ of lacunar infarcts [23].

Limb weakness was more frequent in lacunar syndrome not due to lacunar infarct than in lacunar infarction because non-lacunar infarcts or intracerebral haemorrhages are likely to be more extensive that lacunes [3].
Sudden stroke onset was also more common in patients with lacunar syndrome not due to lacunar infarct. Patients with lacunar infarcts usually present a gradual onset of a focal neurological deficit [24,25], which is in contrast to cardioembolic stroke and also some cases of intraparenchymatous haemorrhages [26] characterised by sudden onset (minutes) of symptoms and maximal neurological deficit at onset. Patients with lacunar syndrome not due to lacunar infarct were younger than those with lacunar infarction, which agrees with other series previously reported [3,27].

It should be noted that a differentiation between patients with single infarcts and patients with multiple infarcts was not performed, nor the presence of coexistent white matter lesions was assessed. In these cases, MRI is the most adequate neuroimaging technique but only $41 \%$ of patients were studied by MRI within this first week of hospital admission. Limitations of the present findings include the single-center characteristics of the study and the fact that this was not a population-based study, as a result of which care should be taken to make inferences to other target populations beyond the subjects in the study.

\section{Conclusions}

Although lacunar syndromes are highly suggestive of small deep cerebral infarctions, lacunar syndromes not due to lacunar infarcts are found in $16.6 \%$ of cases. The presence of sensorimotor stroke, limb weakness and sudden onset in a patient with atrial fibrillation should alert the clinician to the possibility of a lacunar syndrome not due to a lacunar infarct.

\section{List of abbreviations}

CT: Computed tomography; MRI: Magnetic resonance imaging; OR: Odds ratio; SD: standard deviation.

\section{Competing interests}

The authors declare that they have no competing interests.

\section{Authors' contributions}

AA was the principal investigator, designed the study, diagnosed and took care of thepatients, contributed to analyze the data, interpreted the results, wrote the paper, andprepared the final draft. He was alsoresponsible for editorial decisions includingthe selection of the journal.

LGE was the statistician, participated in the study design, analysis andinterpretation of data, wrote the part of thepaper related to the statistical analysis, andapproved the final draft.

$J M, C T, E C$ and OP participated in the collectionof data, medical care of the patients, analysis of results, review of the manuscript for intellectual content, and approved the final draft.

\section{Acknowledgements}

We thank Drs Miquel Balcells, Montserrat Oliveres, Guillermo Arbe, Josep Maria Vives and Adela Vicens for the care of many of the patients included in the study, and Dr Marta Pulido for editing the manuscript and editorial assistance. The study was supported by a grant from FIS PI081514, Madrid, Spain. 


\section{Author Details}

'Unit of Cerebrovascular Diseases, Service of Neurology, Hospital Universitari del Sagrat Cor, Universitat de Barcelona, Barcelona, Spain, 2 Unit of Organization, Planning and Information Systems, Consorci Sanitari del Maresme, Barcelona, Spain, 3Service of Pneumology, Hospital Universitat de Barcelona, Barcelona, Spain and ${ }^{4}$ CIBER de Enfermedades Respiratorias (CB06/ 06), Instituto Carlos III, Madrid, Spain

Received: 19 November 2009 Accepted: 18 May 2010

Published: 18 May 2010

\section{References}

1. Fisher CM: Lacunar strokes and infarcts, a review. Neurology 1982 32:871-876

2. Fisher CM: Lacunar strokes and infarcts, a review. Cerebrovasc Dis 1991 1:311-320.

3. Arboix A, Martí-Vilalta JL: Lacunar syndromes not due to lacunar infarcts. Cerebrovasc Dis 1992, 2:287-292

4. Arboix A, Martí-Vilalta JL: Lacunar stroke. Expert Rev Neurother 2009, 9:179-196.

5. Lodder J, Bamford J, Kappelle J, Boiten J: What causes false clinical prediction of small deep infarcts? Stroke 1994, 25:86-91.

6. Arboix A, Morcillo C, García-Eroles L, Massons J, Oliveres M, Targa C: Different vascular risk factor profiles in ischemic stroke subtypes. The Sagrat Cor Hospital of Barcelona Stroke Registry. Acta Neuro/ Scand 2000, 102:264-270.

7. Special Report from the National Institute of Neurological Disorders and Stroke: Classification of cerebrovascular diseases. III. Stroke 1990, 21:637-676.

8. Arboix A, López-Grau M, Casanovas L, García-Eroles L, Massons J, Balcells M: Clinical study of 39 patients with atypical lacunar syndrome. $J$ Neurol Neurosurg Psychiatry 2006, 77:381-384

9. Silver FL, Norris JW, Lewis AJ, Hachinski VC: Early mortality following stroke: a prospective review. Stroke 1984, 15:492-496

10. Weisberg LA: Computed tomography and pure motor hemiparesis. Neurology 1979, 29:490-495.

11. Stapf C, Hofmeister C, Hartmann A, Marx P, Mast H: Predictive value of clinical lacunar syndromes for lacunar infarcts on magnetic resonance brain imaging. Acta Neuro/ Scand 2000, 101:13-18.

12. Lindgren A, Staaf G, Geijer B, Brockstedt S, Stahlberg F, Holtas S, Norrving B: Clinical lacunar syndromes as predictors of lacunar infarcts. A comparison of acute clinical lacunar syndromes and findings on diffusion-weighted MRI. Acta Neuro/ Scand 2000, 101:128-134.

13. Wessels T, Rottger C, Jauss M, Kaps M, Traupe H, Stolz E: Identification of embolic stroke patterns by diffusion-weighted MRI in clinically defined lacunar stroke syndromes. Stroke 2005, 36:757-761.

14. Arboix A, García-Eroles L, Massons J, Oliveres M, Targa C: Hemorrhagic lacunar stroke. Cerebrovasc Dis 2000, 10:229-234.

15. Mohr JP, Kase CS, Meckler MD, Fisher CM: Sensorimotor stroke due to thalamocapsular ischemia. Arch Neurol 1977, 34:739-741.

16. Blecic S, Bogousslavsky J, van Melle G, Regli F: Isolated sensorimotor stroke, a reevaluation of clinical, topographic and etiological patterns. Cerebrovasc Dis 1993, 3:357-363.

17. Arboix A, Oliveres M, García-Eroles L, Comes E, Balcells M, Targa C: Risk factors and clinical features of sensorimotor stroke. Cerebrovasc Dis 2003, 16:448-451

18. Staaf G, Samuelsson M, Lindgren A, Norrving B: Sensorimotor stroke; clinical features, MRI findings, and cardiac and vascular concomitants in 32 patients. Acta Neurol Scand 1998, 97:93-98.

19. Mead GE, Lewis SC, Wardlaw JM, Dennis MS, Warlow CP: Should computed tomography appearance of lacunar stroke influence patient management? J Neurol Neurosurg Psychiatry 1999, 67:682-684.

20. Cacciatore A, Russo LS Jr: Lacunar infarction as an embolic complication of cardiac and arch angiography. Stroke 1991, 22:1603-1605.

21. Lodder J, Bamford JM, Sandercock PAG, Jones LN, Warlow CP: Are hypertension or cardiac embolism likely causes of lacunar infarction? Stroke 1990, 21:375-381.

22. Fisher CM, Curry HB: Pure motor hemiplegia of vascular origin. Arch Neurol 1965, 13:30-44

23. Arboix A, Martí-Vilalta JL: New concepts in lacunar stroke etiology, the constellation of small-vessel arterial disease. Cerebrovasc Dis 2004 17(Suppl 1):58-62.
24. Benavente $\mathrm{O}$, White $\mathrm{CL}$, Roldan $\mathrm{AM}$ : Small vessel strokes. Current Cardiology Reports 2005, 7:23-28.

25. Arauz A, Murillo L, Cantu C, Barinagarrementeria F, Higuera J: Prospective study of single and multiple lacunar infarcts using magnetic resonance imaging risk factors, recurrence, and outcome in 175 consecutive cases. Stroke 2003, 34:2453-2458.

26. Petty GW, Brown RD Jr. Whisnant JP, Sicks JD, O'Fallon WM, Wiebers DO: Ischemic stroke subtypes: a population-based study of functional outcome, survival, and recurrence. Stroke 2000, 31:1062-1068.

27. Lewington S, Clarke R, Qizilbash N, Peto R, Collins R: Age-specific relevance of usual blood pressure to vascular mortality: a metaanalysis of individual data for one million adults in 61 prospective studies. Lancet 2002, 360:1903-1913.

\section{Pre-publication history}

The pre-publication history for this paper can be accessed here: http://www.biomedcentral.com/1471-2377/10/31/prepub

doi: 10.1186/1471-2377-10-31

Cite this article as: Arboix et al., Clinical predictors of lacunar syndrome not due to lacunar infarction BMC Neurology 2010, 10:31

\section{Submit your next manuscript to BioMed Central and take full advantage of:}

- Convenient online submission

- Thorough peer review

- No space constraints or color figure charges

- Immediate publication on acceptance

- Inclusion in PubMed, CAS, Scopus and Google Scholar

- Research which is freely available for redistribution

Submit your manuscript at www.biomedcentral.com/submit
C) Biomed Central 